\title{
Effective Reservoir Management models leading to Business Decisions
}

\author{
Speaker Mike King (Department of Petroleum Engineering, Texas A\&M University)
}

Reservoir management can be thought of as a sequence of activities in which we:

1) Build a model, or class of models, that embodies our understanding of the reservoir description and reservoir processes to obtain predictions of how the field will perform

2) Use these models to make a business decision, e.g., the placement of an infill well

3) Measure the reservoir performance after the action is taken, and either revise or validate the models being used for subsequent predictions

Each step of this process can be enhanced through the use of multiple models. When we work with more than a single model we're better able to represent our uncertainty in performance prediction and to expose the risk associated with the business decision. We are also better able to design a surveillance program by attempting to distinguish between different subsurface models. Examples will be drawn from North Sea experience with mature assets on the success (or failure) of these strategies. 